\title{
Über die nach den Methoden der Lecithindarstellung aus Pflanzensamen erhältlichen Verbindungen.
}

\author{
II. Mitteilung. \\ Vergleichende Hydrolyse von Bilecithin. \\ Von \\ Georg Trier.
}

(Aus dem agrikultur-chemischen Laboratorium der Eidgenössischen Technischen Hochschule in Zürich.)

(Der Redaktion zugegangen am 10. Juni 1913.)

Nachdem ich festgestellt hatte, daß bei der Hydrolyse von Bohnensamenlecithin Aminoäthylalkohol auftritt, suchte ich diese Verbindung auch in anderen Lecithinen nachzuweisen. Da mir nun aber zunächst keine andern Lecithinpräparate zur Verfügung standen, mußte ich $\mathrm{zu}$ käuflichem Eilecithin greifen. Gleichzeitig verband ich damit den Vergleich der Gewinnung von Glycerinphosphorsäure aus Pflanzenlecithin und Eilecithin, um der Ursache nachzugehen, weshalb die Gewinnung reiner Präparate von glycerinphosphorsaurem Baryum aus pflanzlichen Phosphatiden sich so schwierig gestaltet.

Die Angaben über die stickstoffhaltigen Bestandteile des Eilecithins lauten widersprechend. Angaben von quantitativen Ausbeuten an Cholin finden sich in der Dissertation von Lüdecke, ${ }^{1}$ ) ferner in einer Arbeit von Malengreau und Prigent. ${ }^{2}$ ) Letztere Forscher haben, ebenso wie mehrere Vorgänger, das Cholin als Platinat zur Wägung gebracht. Sie verwendeten das Eilecithin Kahlbaum und reinigten es über die Cadmiumverbindung. Sie erhielten dann nach mehrstündiger Hydrolyse mit n/10-Schwefelsäure 95-96\% des Gesamtstickstoffs als Cho-

1) Lüdecke, Zur Kenntnis der Glycerinphosphorsäure und des Lecithins. Dissert. München 1905.

2) Malengreau u. Prigent, Diese Zeitschr., Bd. 77, S. 107 (1912). 
linplatinat wieder. Der Stickstoffgehalt ihres Präparates betrug aber nur $1,385 \%$. Lüdecke bezeichnet die Bestimmung des Cholins als Platinat als nicht geeignet und wäblt einen eigenartigen Weg, der Spaltung des Lecithins mit Pikrinsäure und Wügung des Cholins als Pikrat. Er erhielt 15,05\% Cholin an Stelle der für das aideelle Lecithin * berechneten $15,53 \%$, doch enthielt sein Präparat, welches ebenfalls über die Cadmiumverbindung gereinigt worden war, $2,4 \% \mathrm{~N}$ an Stelle der für das aideelle Lecithin berechneten $1,8 \% \mathrm{~N}$.

Andere Untersucher haben nur kleinere Anteile des Gesamtstickstoffs für das Cholin reklamiert. So gibt Mac Lean ${ }^{1}$ ) für Eilecithin $66 \%$, für Handelslecithin $80 \%$, für die Cadmiumverbindung $75 \%$ an.

Andere Stickstoffverbindungen als Cholin sind meines Wissens aus Eilecithin nicht isoliert worden. Lüdecke fand, daß die Fettsäuren reichlich Stickstoff enthalten, und führt dies auf den Umstand zurück, daß bei der Spaltung der Cadmiumverbindung mit Ammoncarbonat sich Ammoniak an ungesättigte Fettsäuren anlagern könnte, woraus sich auch der große Stickstoffgehalt seines Präparats erklären würde. Es ist in der Tat auffällig, daß die aus den Cadmiumverbindungen erhaltenen Lecithine oft (Ausnahmen siehe bei Malengreau und Prigent, Mac Lean) stickstoffreicher werden als das Ausgangsmaterial.

Es ist aber nicht zu bezweifeln, daß auch nicht über die Cadmiumverbindung gereinigte Präparate Stickstoff in der Fettsäurenfraktion gebunden enthalten, der auch bei langdauernder Hydrolyse und vollkommenem Auswaschen nicht entfernt werden kann. (Siehe bei Mac Lean.)

Für meine Zwecke war eine Reinigung des Handelspräparates weder notwendig, noch erwünscht. Über das von mir zunächst verwendete Präparat von E. Merck, Darmstadt, urteilt J. Bang, ${ }^{2}$ ) es sei "sehr unrein und zu wissenschaftlichen Zwecken vollkommen unbrauchbar » da es $2,05 \% \mathrm{~N}$ und $3,35 \% \mathrm{P}$ enthalte. Das von mir untersuchte Präparat enthielt indessen $1,85 \% \mathrm{~N}$ und $3,67 \% \mathrm{P}$.

1) Mac Lean, Diese Zeitschr, Bd. 59, S. 223 (1909).

2) J. Bang, Chemie und Biochemie der Lipoide. Wiesbaden 1911. 
0,7382 g Substanz gaben $0,0971 \mathrm{~g} \mathrm{Mg}_{2} \mathrm{P}_{2} \mathrm{O}_{7}=3,66 \% \mathrm{P}$ 0,6528 » " 0,0863 » $=3,67 \% \mathrm{P}$ 0,5098 g Substanz gaben nach Kjeldahl 0,00974 g N=1,91\% N

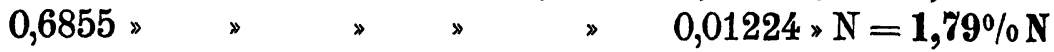
0,8591 * $\quad$ " $\quad 0,01603$ » $\mathrm{N}=1,86 \% \mathrm{~N}$

Das Verhältnis von $P: N$ ist gleich $1: 1,12$; es ist nahezu 1:1, wenn man vom Gesamtstickstoff jenen abzieht, der im Fettsäurenrückstand verbleibt und welcher offenbar zum $P$ in keiner Relation steht. Auf diese Sachlage hat bereits Mac Le an hingewiesen.

Das Präparat enthielt 3,5\% Feuchtigkeit, auf welche bei nicht besonders getrockneten Portionen in der Berechnung Rücksicht genommen wurde.

Nach einigen Vorversuchen wurden $81,20 \mathrm{~g}$ des Lecithins entsprechend 78,34 $\mathrm{g}$ Trockensubstanz mit $500 \mathrm{ccm} 5 \%$ iger Schwefelsäure 6 Stunden unter Rückflußkühlung hydrolysiert. Das von den gut ausgewaschenen Fettsäuren getrennte Filtrat wurde wiederholt mit Äther ausgeschüttelt, der Äther entfernt, die Lösung wieder auf $500 \mathrm{ccm}$ gebracht und mit konzentrierter Phosphorwolframsäure ausgefällt.

Die Fällung wurde mittels Baryt in bekannter Weise zerlegt, die erhaltene Basenlösung mit überschüssiger Salzsäure versetzt und auf $200 \mathrm{ccm}$ gebracht.

1. $10 \mathrm{ccm}$ dieser Lösung enthielten $0,0509 \mathrm{~g} \mathrm{~N}$

2. 10 » » 0,0506 »

Im Mittel also $0,1015 \mathrm{~g} \mathrm{~N}$ in $20 \mathrm{ccm}$ oder $1,015 \mathrm{~g} \mathrm{~N}$ in der ganzen Fällung; dies entspricht $70 \%$ des gesamten Stickstoffs. ${ }^{1)}$ Aus der eingedunsteten Lösung krystallisierte das salzsaure Cholin in mehrere Zentimeter langen schön ausgebildeten Nadeln. Schon diese Art des Krystallisierens spricht für große Reinheit des Cholins. Durch Fällung mit alkoholischer Sublimatlösung wurde das Cholin in das Quecksilberdoppelsalz übergeführt und im Filtrat der Fällung nach weiteren Basen gesucht. Solche waren nur noch spurenweise vorhanden, Colamin

1) Unter den eingehaltenen Bedingungen dürfen 5-10\% des Cholins der Fällung entgangen sein. 
konnte hier nicht nachgewiesen werden. Es wurde aus der verdünnten Lösung durch Phosphorwolframsäure nicht gefällt.

Das Filtrat von der Phosphorwolframsäurefällung wurde auf $600 \mathrm{ccm}$ gebracht:
1. $50 \mathrm{ccm}$ enthielten $0,02138 \mathrm{~g} \mathrm{~N}$
2. $50,0,02069, \mathrm{~N}$

In $100 \mathrm{ccm}$ waren also $0,04207 \mathrm{~g} \mathrm{~N}$ vorhanden, im ganzen Filtrat 0,2524 g N, entsprechend 17,42\% des Gesamtstickstoffs.

Das Filtrat wurde mittels Baryt von den unorganischen Säuren (Schwefelsäure, Phosphorsäure, Phosphorwolframsäure) befreit, der überschüssige Baryt durch Kohlensäure entfernt und die Lösung stark eingeengt. Dabei schied sich das in heißem Wasser schwer lösliche Baryumsalz der Glycerinphosphorsäure in rein weißen Flocken aus. Es wurde dann wieder in Lösung gebracht und mit einem Überschuß von $95 \%$ igem Alkohol ausgefällt. Die Fällung wurde mit Alkohol und Äther gewaschen; sie trocknete zu einem schneeweißen leicht verreiblichen Pulver ein. In dieser einfachen Weise ließen sich gleiche Präparate von glycerinphosphorsaurem Baryum aus pflanzlichem Material nicht darstellen. Damit ist also sichergèstellt, daß es nicht in mangelhafter Darstellungstechnik liegt, wenn man aus Pflanzenlecithinen zunächst nur schwer trocknende, gefärbte und ungemein hygroskopische Baryumsalze erhält. Es muß vielmehr im chemischen Bau dieser Lecithine liegen, daß sie bei gleicher Behandlung ungleiche Präparate liefern.

Das Filtrat von der Alkoholfällung wurde angedunstet, nach Befreiung vom Alkohol zwecks weiterer Reinigung mit Bleiessig gefällt. ${ }^{1}$ ) . Das Filtrat von dieser Fällung wurde mittels Schwefelwasserstoff entbleit; dann mußte noch von etwas vorhandenem Cholin durch Fällen mit alkoholischer Sublimatlösung befreit werden. Nach Entfernung des Quecksilbers wurde bei Gegenwart 5\% iger Schwefelsäure wieder mit Phosphorwolfram-

1) Hier wird noch ein Teil der Glycerinphosphorsäure gefällt. Durch die Behandlung mit Kohlensäure bei Gegenwart der alkoholischen Basen (Cholin, Colamin) wird die Glycerinphosphorsäure zum Teil an diese gebunden und entgeht der Alkoholfällung. 
säure, diesmal aber in ganz konzentrierter Lösung, gefällt. Die aus der Fällung regenerierten Basen wurden mit starker Salzsäure eingedunstet uud mit Goldlösung versetzt. Es entstand keine Fällung, nach einigem Stehen aber Krystallisation von gut ausgebildeten Colaminauratkrystallen.

Eine erste Krystallisation dieses Aurats gab 49,25\% Au und $35,20 \%$ Cl. ${ }^{1}$ ).

Berechnet für Colaminaurat $\mathrm{C}_{2} \mathrm{H}_{8} \mathrm{ONAuCl}_{4} 49,17 \%$ Au und $35,36 \% \mathrm{Cl}$.

Das Goldsalz schmolz bei $188-190^{\circ}$ ohne Zersetzung. Auch aus dem Filtrat der Phosphorwolframfällung ließ sich noch eine kleine Menge gut ausgebildeter Colaminauratkrystalle gewinnen, aber erst nach mehrfacher Umkrystallisation und Entfernung von ausgeschiedenem Gold.

\section{$0,1377 \mathrm{~g}$ Goldsalz gaben $0,0678 \mathrm{~g} \mathrm{Au}=49,24 \% \mathrm{Au}$.} Schmelzpunkt des Aurats 186-190\%.

Aus der beschriebenen Hydrolyse war zu entnehmen, daß auch im Eilecithin eịn Teil der Alkoholbase in nichtmethylierter Form vorliegt. Die Ausbeute an Aminoäthylalkohol (Colamin) war aber sehr gering, was an der sehr umständlichen Darstellungsweise zu liegen schien. In einem weiteren Versuch wurde nun von der Verwendung der Phosphorwolframsäure, wie von der Isolierung anderer Spaltungsprodukte abgesehen. Die Ausbeute stieg dann sogleich auf ein Vielfaches. Es wurden $100 \mathrm{~g}$ des Merck schen Lecithins mit $1121 / 2 \%$ iger Schwefelsäure hydrolysiert. Die $A r t,{ }^{2}$ ) wie verfahren wurde, habe ich schon mitgeteilt. ${ }^{3}$ ) Es wurden dann ohne Schwierigkeit 2,5 g schön ausgebildeter Krystalle des Colaminaurats erhalten. Sie schmolzen ohne Zersetzung bei $188-190^{\circ}$.

Später erhielt ich eine größere Menge eines anderen Eilecithins. Dieses Präparat enthielt 3,46\% P und 1,66\% N.

1) Diese Zeitschrift, Bd. 76, S. 498 (1912).

2) Das Filtrat von den Fettsäuren wurde mit Baryt und Bleiessig gereinigt, dann das Cholin durch Fällen mit Sublimat und Platinchlorid vollkommen entfernt.

3) Diese Zeitschr., Bd. 76, S. 496 (1912). 
1. $1,1179 \mathrm{~g}$ gaben $0,1422 \mathrm{~g} \mathrm{Mg}_{2} \mathrm{P}_{2} \mathrm{O}_{7}=3,54 \% \mathrm{P}$

2. $1,1674: \Rightarrow 0,1438, \rightarrow=3,42 \% \mathrm{P}$

3. $1,6561,, 0,2034,,=3,42 \% \mathrm{P}$

1. 1,$4457 ;, 0,02408 \mathrm{~g} \mathrm{~N}=1,67 \% \mathrm{~N}$

2. 1,2766 * $0,02124 \times \mathrm{N}=1,66 \% \mathrm{~N}$

In diesem Präparat wurde die Menge des Colamins mittels des Apparats von van Slyke bestimmt und die Art seiner Bindung im Lecithinmolekül festgestellt.

2,6140 $\mathrm{g}$ des Lecithins wurden erst mit verdünnter Kalilauge gespalten, dann mit verdünnter Schwefelsäure eine Stunde gekocht. Das Filtrat von den Fettsäuren wurde auf $50 \mathrm{ccm}$ gebracht.

1. $11,6 \mathrm{~cm}$ gaben $2,4 \mathrm{ccm} \mathrm{N}$ bei $18{ }^{\circ}$ und $714 \mathrm{~mm}=0,21 \%$ Aminostickstoff

2. $18,95,>3,4,>>$, , > $=0,18 \%$ "

3. $9,65,, 2,0,>,>,>,=0,21 \%$,

Im Mittel wurden $0,20 \%$ Aminostickstoff erhalten.

$7,408 \mathrm{~g}$ Lecithin wurden mit $200 \mathrm{ccm} 1 \%$ iger Schwefelsäure 8 Stunden hydrolysiert, das Filtrat von den Fettsäuren auf $60 \mathrm{ccm}$ gebracht.

1. $9,15 \mathrm{ccm}$ gaben $4,04 \mathrm{ccm} \mathrm{N}$ bei $17^{\circ}$ und $721 \mathrm{~mm}=0,20 \%$ Aminostickstoff

2. $9,25:, 4,8>>>,>>>=0,23 \%$,

Im Mittel wurden 0,21\% Aminostickstoff erhalten. Man könnte daraus folgern, daß das Lecithin 0,205\% Colaminstickstoff oder 0,89\% Colamin enthalte. Dieser Schluß ist aber, wie weitere Bestimmungen zeigen, noch keineswegs erlaubt. Bei der Spaltung mit Baryt geht nämlich nur etwa die Hälfte, also 0,1\% Aminostickstoff in das Filtrat von den Baryumverbindungen, und ähnliche Verhältnisse zeigten auch die anderen in gleicher Richtung untersuchten Präparate (aus Hafersamen und Erbsensamen). Nur von dem bei der Barytspaltung in das Filtrat übergehenden Aminostickstoff darf man vorläufig annehmen, daß er dem vorhandenen Colamin entspricht. Von jenem Teil des Aminostickstoffs, der erst bei der Säurehydrolyse ins Filtrat von den Fettsäuren gelangt, läßt sich vorläufig nicht angeben, ob er einer anderen Verbindung angehört oder einem Teil "des Colamins, welches im Lecithin in einer nur durch Säuren abhydrolysierbaren Form vorliegt. 
Ein Teil des Lecithins wurde in Äther gelöst und mit einer verdünnten Lösung von Natriumnitrit und verdünnter Essigsäure im Scheidetrichter gut durchgeschüttelt. Hierauf wurde durch wiederholtes Auswaschen die salpetrige Säure ganz entfernt. Die vollständige Trennung der Schichten im Scheidetrichter ließ sich durch Zugabe von Kochsalz und Alkohol bewirken. Das Lecithin wurde dann aus der alkoholischätherischen Lösung nach bekanntem Verfahren wiedergewonnen. Von dem so behandelten Lecithin wurden $1,367 \mathrm{~g}$ mit $60 \mathrm{ccm}$ $1 \%$ iger Schwefelsäure 71/2 Stunden unter Rückflußkühlung gekocht.

Das Filtrat von den Fettsäuren wurde auf $25 \mathrm{ccm}$ gebracht. Eine Probe gab sowohl mit Phosphorwolframsäure, wie mit Wismutnatriumjodid starke Fällung; es waren also die Basen (Cholin) bei der Behandlung mit salpetriger Säure nicht entfernt worden.

1. $9,55 \mathrm{ccm}$ gaben $1,2 \mathrm{ccm} \mathrm{N}$ bei $222^{\jmath}$ und $714 \mathrm{~mm}=0,12 \%$ Aminostickstoff 2. $11,45,>1,5>18^{\circ}, 716>=0,13 \%$,

Im Mittel wurden also 0,12.5 $\%$ Aminostickstoff gefunden, während das unveränderte Lecithin bei der Säurespaltung $0,20 \%$ Aminostickstoff geliefert hatte. Die Differenz entfällt auf den Stickst off des Colamins. Bei der Hydrolyse mit Baryt wurde auch tatsächlich diese Differenz $(0,09 \%$ Aminostickstoff, siehe unten) erhalten. Ferner ließ sich zeigen, daß diese Aminostickstoffmenge wesentlich dem Colamin angehört. Damit scheint mir als o bewiesen, daß das bei der Barythydrolyse freiwerdende Colamin im Lecithin vermittelst der Hydroxylgruppe gebunden ist, während die A minogruppe frei ist. (Die Bestimmung der freien Aminogruppen im Lecithin selbst habe ich auch versucht, doch läßt sich das weniger gut ausführen.)

In einem weiteren Versuch wurde das Lecithin in ähnlicher Weise mit salpetriger Säure behandelt, dann aber mit Baryt gespalten. Die Menge des Aminostickstoffs ging, wie zu erwarten war, auf ein Minimum zurück.

10,67 g Lecithin wurden, wie oben beschrieben, im Scheidetrichter mit salpetriger Säure behandelt, die ausgewaschene alkoholisch-ätherische Lecithinlösung sodann in heiße 
Barytlösung einfließen gelassen und 1 Stunde am siedenden Wasserbade belassen. Es wurde dann von den gut ausgewaschenen Baryumverbindungen getrennt, mit Schwefelsäure vom Baryt befreit und auf $70 \mathrm{ccm}$ gebracht.

1. $20 \mathrm{ccm}$ gaben $0,9 \mathrm{ccm} \mathrm{N}$ bei $20^{\circ}$ und $725 \mathrm{~mm}$

2. $20>>1,1>>>$ " " "

Im Mittel $1,0 \mathrm{ccm} \mathrm{N}=0,01_{8} \%$ Aminostickstoff. $20 \mathrm{ccm}$ enthielten noch $0,0320 \mathrm{~g} \mathrm{~N}=1,05 \% \mathrm{~N}$.

Der Gesamtstickstoff war also nur um wenig vermindert (siehe unten), die Lösung gab auch sehr starke Fällungen mit Phosphorwolframsäure und Natriumwismutjodid (Cholin). Dagegen war der Colaminstickstoff praktisch verschwunden.

Von der Annahme ausgehend, daß die Fällbarkeit des Lecithins durch Metallsalze. wie Platin- und Cadmiumchlorid wesentlich eine Funktion der quaternären Base (Cholin) ist, prüfte ich, inwieweit die Behandlung mit Cadmiumchlorid eine Trennung des Cholin-Lecithins vom Colamin-Lecithin ermöglicht. Es wurde das Verhältnis vom Gesamtstickstoff zum Colaminstickstoff nach der Barythydrolyse ermittelt und konstatiert, da $B$ sich im Filtrat von der Cadmiumfällung das ColaminLecithin anhäuft.

2,580 g Lecithin wurde mit überschüssigem Baryt eine Stunde am kochenden Wasserbad zersetzt. Gleichzeitig wurde auch die in alkoholischer Lösung mit alkoholischem Cadmiumchlorid erhaltene Fällung, sowie das Filtrat dieser Fällung samt Waschalkohol mit Barytwasser in gleicher Weise zersetzt. Zur Kontrolle wurde auch ein aliquoter Teil der alkoholischen Lecithinlösung vor der Fällung mit Cadmium in gleicher Weise hydrolysiert. Die von den gut ausgewaschenen Rückständen getrennten Lösungen wurden mittels Kohlensäure vom überschüssigen Baryt befreit und dann auf ein bestimmtes Volumen gebracht.

Das Hydrolysat von den 2,580 Lecithin wurde auf $60 \mathrm{ccm}$ gebracht:

1. $20 \mathrm{ccm}$ gaben $0,00946 \mathrm{~g}$ Gesamtstickstoff $=1,10 \% \mathrm{~N}$

2. $20 \mathrm{ccm}$ gaben im van Slykeschen Apparat $1,4 \mathrm{ccm} \mathrm{N}$ bei $20^{\circ}$ und $724 \mathrm{~mm}=0,76 \mathrm{mg}$ Aminostickstoff $=0,09 \%$. 
Das Verhältnis des Gesamtstickstoffs zum Aminostickstoff beträgt etwa $12: 1$.

Der aliquote Teil der alkoholischen Lecithinlösung, die nachher mit Cadmium gefällt wurde, 2,5 g Lecithin entsprechend, gab ein Hydrolysat, das auf $80 \mathrm{ccm}$ gebracht wurde.

1. $20 \mathrm{ccm}$ gaben $0,00705 \mathrm{~g}$ Gesamtstickstoff $=1,11 \% \mathrm{~N}$

2. $20 \gg, 1,0 \mathrm{ccm} \mathrm{N}$ bei $20^{\circ}$ und $724 \mathrm{~mm}$

3.20 » 1,0 , $>20$ " 724 , $=0,54 \mathrm{~g}$ Aminostickstoff $=0,09 \%$.

Gesamtstickstoff: Aminostickstoff $=12 ; 1$.

Das Hydrolysat der Cadmiumfällung wurde auf $90 \mathrm{ccm}$ gebracht:

1. $20 \mathrm{ccm}$ gaben $0,01135 \mathrm{~g}$ Gesamtstickstoff.

2. $20 \gg, 0,01126 \mathrm{~g}$

$3.30 » \quad 1,7 \mathrm{~cm} \mathrm{~N}$ bei $20^{\circ}$ und $724 \mathrm{~mm}=0,92 \mathrm{mg}$ Aminostickstoff.

Die Cadmiumfällung enthielt daher im ganzen $0,05085 \mathrm{~g}$ Stickstoff, davon $0,00276 \mathrm{~g}$ Aminostickstoff. Das Verhältnis ist $18,4: 1$.

Das Hydrolysat vom Filtrat der. Cadmiumfällung wurde auf $50 \mathrm{ccm}$ gebracht:

1. $20 \mathrm{ccm}$ enthielten 0,00198 Gesamtstickstoff

2. $9,05 \mathrm{ccm}$ gaben $0,6 \mathrm{~cm} \mathrm{~N}$ bei $20^{\circ}$ und $724 \mathrm{~mm}$

3. $20 \mathrm{~cm} 1,2$, $\mathrm{N}$, " " ,

Das Filtrat der Cadmiumfällung enthielt also bei $0,00495 \mathrm{~g}$ Gesamtstickstoff $0,00172 \mathrm{~g}$ Aminostickstoff. Das Verhältnis ist hier $2,9: 1$.

Der nun folgende Versuch hatte vornehmlich den Zweck, die bei der Barytspaltung isolierbare Colaminmenge mit der aus den Bestimmungen nach van Slyke berechneten zu vergleichen.

$100 \mathrm{~g}$ Eilecithin wurden mit überschüssiger Barytlösung 3 Stunden am kochenden Wasserbad zerlegt. Das Lecithin wurde in ätherischer Lösung langsam einfließen gelassen. Durch mechanische Rührung wurde für feinste Verteilung gesorgt. Nach vollkommenem Auswaschen der Baryumverbindungen wurde mittels Kohlensäure der überschüssige Baryt entfernt,

Hoppe-Seyler's Zeitschrift f. physiol. Chemie. LXXXVI. 
dann mit Bleiessig gefüllt, von der ausgewaschenen Fällung durch Filtration getrennt, mit Schwefelwasserstoff entbleit und das Filtrat vom Bleisulfid auf $200 \mathrm{ccm}$ gebracht.

1. $5 \mathrm{ccm}$ enthielten $0,02236 \mathrm{~g} \mathrm{~N}=0,89 \% \mathrm{~N}$ (auf das Lecithin berechnet)

2. 5, $0,02193 \mathrm{~g} \mathrm{~N}=0,88 \% \mathrm{~N}$

3. 7,7 gaben $6,4 \mathrm{ccm} \mathrm{N}$ bei $20^{\circ}$ und $720 \mathrm{~mm}$ (nach van Slyke) $=0,09 \%$ Aminostickstoff

4. $9,3 \mathrm{~cm}$ gaben $7,4 \mathrm{~cm} \mathrm{~N}$ bei $20^{\circ}$ und $720 \mathrm{~mm}=0,09 \%$ Aminostickstoff

$10 \mathrm{ccm}$ wurden mit $0,3 \mathrm{ccm}$ Schwefelsäure versetzt und mit konzentrierter Phosphorwolframsäure gefällt. Die Fällung ist sehr voluminös und breiig. Sie enthielt $0,0414 \check{\mathrm{g} \mathrm{N}}=$ $0,83 \% \mathrm{~N}$.

Das Filtrat der Fällung enthielt 0,00327 $\mathrm{N}=0,06 \% \mathrm{~N}$.

Die Phosphorwolframfällung zieht viel Wasser an sich, die Lösung wird dadurch so konzentriert, daß auch das Colamin zum Teil ausfällt. Bei Gegenwart von viel Cholin wird daher das Colamin auch aus Lösungen gefällt, deren Gehalt an Colamin geringer ist, als seiner ermittelten Fällbarkeit entspricht.

(Der Gehalt der Lösung an Colamin betrug hier nur etwa $0,04 \%$.)

$150 \mathrm{ccm}$ des Filtrats wurden nun weiter auf Basen verarbeitet. Es wurde in salzsaures Salz übergeführt, durch Behandeln mit absolutem Alkohol von Baryumchlorid getrennt. Die organischen Chloride waren in Alkohol leicht löslich. Dann wurde in bekannter Weise mit Sublimat das Cholin entfernt. Die Quecksilberfällung enthielt ausschlieBlich Cholin. Ein aliquoter Teil der Quecksilberfällung wurde in das salzsaure Salz übergeführt, welches schön krystallisierte und, mit Goldlösung gefällt, bis in die allerletzten Fraktionen reines Cholinaurat enthielt. 1. Krystallisation: $0,2805 \mathrm{~g}$ Aurat gaben $0,1244 \mathrm{~g} \mathrm{Au}=\mathbf{4 4 , 3 6} \% \mathrm{Au}$ Aus der Mutterlauge gab eine

2. Krystallisation: 0,4868 g Aurat $\ldots 0,2160 \mathrm{~g} \mathrm{Au}=44,37 \% \mathrm{Au}$ 3. Krystallisation: $0,3125 \mathrm{~g}$ Aurat $\ldots 0,1388 \mathrm{~g} \mathrm{Au}=\mathbf{4 4 , 4 2 \% ~} \mathrm{Au}$

Aus der Mutterlauge der 3. Krystallisation wurde noch eine kleine Menge einer 4. Krystallisation erhalten, die gleich den anderen Fraktionen beim raschen Erhitzen bei $262^{\circ} \mathrm{schmolz}$.

Aus dem Filtrat der Quecksilberfällung wurde nach Entfernung des Alkohols das Sublimat auskrystallisieren gelassen. 
Filtrat und Waschwässer dieser Ausscheidung dienten zur Gewinnung des Colamins. Die Ausscheidung selbst wurde mit Schwefelwasserstoff zersetzt und geprüft, ob sie noch organische Basen einschließe. Es wurde eine minimale Menge eines Chloraurats erhalten, das in feinen Nädelchen sich ausschied, erst sehr unscharf zwischen 150-160 stallisieren aus starker Salzsäure gegen $190^{\circ}$ schmolz und somit wahrscheinlich nur aus einem kleinen Rest hier zurückgebliebenen Colamins bestand. Es ließen sich also auch hier die Zwischenprodukte der Methylierung von Colamin zum Cholin nicht nachweisen.

Die vom Quecksilber befreite Colaminfraktion wurde eingedunstet, mit Wasser aufgenommen, mit Tierkohle ganz entfärbt und dann auf $100 \mathrm{ccm}$ gebracht. Sie sollte nach obiger Bestimmung noch $0,0675 \mathrm{~g}$ Aminostickstoff enthalten.

Gefunden wurden nur noch $0,0594 \mathrm{~g}$.

$10 \mathrm{ccm}$ gaben nach van Slyke $11,0 \mathrm{ccm} \mathrm{N}$ bei $21^{\circ}$ und $726 \mathrm{~mm}=0,00594 \mathrm{~g}$ Aminostickstoff. Außerdem schien noch etwas Stickstoff in anderer Form vorzuliegen, denn $10 \mathrm{ccm}$ gaben $0,00731 \mathrm{~g}$ Gesamtstickstoff.

$50 \mathrm{ccm}$ der Lösung wurden eingedunstet und bei Gegenwart von konzentrierter Salzsäure mit Goldlösung versetzt und krystallisieren gelassen. Diese $50 \mathrm{ccm}$ mit 0,0297 g Aminostickstoff sollten $0,8503 \mathrm{~g}$ Colaminchloraurat liefern. Es schieden sich aus dem dicken Sirup an gut ausgebildeten Krystallen $0,443 \mathrm{~g}$ Colaminaurat aus. Es war also etwas mehr als die Hälfte der berechneten Menge auskrystallisiert. Daß in der glycerinhaltigen sirupösen Lösung überhaupt eine Krystallisation erfolgt, ist nur dem ausgezeichneten Krystallisationsvermögen des Aurats zu danken. Es ist kaum zu bezweifeln, daß die berechnete Colaminmenge hier auch vorhanden war. Die Annahme, daß der bei der Barytspaltung ermittelte Aminostickstoff wesentlich oder ausschließlich auf das Colamin zu beziehen ist, erscheint somit gerechtfertigt.

Nach Umkrystallisation aus starker Salzsäure wurden gut ausgebildete Krystalle erhalten.

$0,2766 \mathrm{~g}$ gaben $0,1356 \mathrm{~g} \mathrm{Au}=49,03 \mathrm{Au}$ 
152 Georg Trier, Aus Pfanzensamen erhältliche Verbindangen. II.

Für Colaminoaurat $\mathrm{C}_{2} \mathrm{H}_{8} \mathrm{ON} \cdot \mathrm{AuCl}_{4}$ berechnet $49,17_{0} / 0 \mathrm{Au}$. Schmelzpunkt $188^{\circ}$.

Von den bei der Barytspaltung ins Filtrat übergegangenen Stickstoffverbindungen konnten nur Colamin und Cholin aufgefunden werden. Das letztere konnte fast quantitativ in Form des Chloraurats gewonnen werden. Nach den quantitativen Bestimmungen enthielt das Filtrat nach der Bleifällung nämlich noch 0,8\% $\mathrm{N}$ als Cholin. Die $200 \mathrm{ccm}$ sollten 6,9 g Cholin geben; aus der Menge des erhaltenen Chloraurats berechnet sich die isolierte Cholinmenge auf $6,6 \mathrm{~g}$. 